Progressive or degressive compression pressure profile in patients with chronic venous disorders of the lower limb

\section{Giovanni Mosti}

\section{Angiology Department, MD Barbantini Clinic, Lucca, Italy}

\section{Abstract}

Graduated compression devices are considered the standard care for management of venous and lymphatic disorders. Recently compression devices exerting a pressure over the calf higher than over the ankle have been proved to be more effective than traditional graduated devices in increasing the impaired ejection fraction (EF) from the lower leg in patients with venous disease. Aim of this work is presenting an overview of the new concept on progressive compression, its potential benefits and limits. In different series of tests, the EF from the lower leg was assessed in 70 patients with severe reflux in the great saphenous vein (GSV). EF was measured by strain gauge plethysmography, in baseline conditions and after applying graduated compression devices or the new inversely graduated or progressive compression (PC) devices. The interface pressure was recorded, simultaneously with the EF, both in the gaiter area (B1 point) and at the calf ( $\mathrm{C}$ point) in order to assess the compression pressure profile. EF, severely impaired in patients with GSV reflux, was increased by compression. So called PC devices (both PC elastic stocking and PC inelastic bandages) were significantly more effective than graduated compression in increasing the ejection fraction. The higher the pressure on the calf the higher the $\mathrm{EF}$ improvement. Maintaining the same strong pressure over the calf by means of two progressive stockings and increasing the pressure only over the calf to restore a graduated compression didn't improve the EF. To improve venous pumping function in the ambulant patient stronger compression of the calf is more effective than graduated compression. This can be explained by the higher amount of blood volume pooled in the calf veins.

\section{Introduction}

Compression providing a graduated pressure profile (higher over the ankle and gradually decreasing from distal to proximal) is the general principle for every kind of compression therapy and has been the standard care for thromboprophylaxis and management of venous and lymphatic disorders so far. This principle of a graduated compression is considered so important that it is mandatory in regulatory standards for elastic compression stockings. ${ }^{1-3}$

Recent publications have questioned the importance of a graduated compression at least in ambulatory patients, both regarding subjective symptoms relief $f^{1-3}$ and improvement of objective parameters. ${ }^{4-6}$

It was clearly shown that, in patients with venous disease, the reduced ejection fraction (EF) from the lower leg can be significantly more increased by compression devices exerting a higher pressure over the calf than over the ankle area compared with compression devices exerting a graduated pressure profile. This is true both for elastic compression stockings ${ }^{4}$ and for inelastic bandages ${ }^{5}$ which are named inversely graduated or progressive compression devices.

The following will give an overview of our results.

\section{Materials and Methods}

In different series of experiments a total of 70 patients ( 29 males and 41 females; mean age of 53.1 years; range $37-70$ ) affected by chronic venous disease were investigated without and with different compression devices. Clinically these patients corresponded to classes $\mathrm{C} 2-\mathrm{C} 5$ in the Clinical, Etiological, Anatomic and Pathophysiologic classification (CEAP).

Inclusion criteria: all patients were affected by insufficiency of the great saphenous vein (GSV) with: terminal and pre-terminal valve incompetence; venous diameter in standing position $2 \mathrm{~cm}$ below the sapheno-femoral junction more than $1 \mathrm{~cm}$; venous reflux time after manual calf compression longer than 3 seconds; able to perform the physical exercise necessary to complete the protocol; with an ankle-brachial pressure index measured by Doppler higher than 0.8 .

Exclusion criteria: patients with competent terminal and/or pre-terminal valves, with a venous diameter in the standing position $2 \mathrm{~cm}$ below the junction less than $1 \mathrm{~cm}$, with a venous reflux time shorter than 3 seconds, those unable to perform the exercise reported in the protocol or with an ankle brachial pressure index lower than 0.8 .

Duplex investigation (Esaote MyLab 60; linear probe 7.5 to $10 \mathrm{MHz}$; Esaote s.p.a., Genoa, Italy) of the superficial and deep veins of the lower extremity was performed with the patient in the standing position for detecting venous reflux during Valsalva's maneuver and
Correspondence: Giovanni Mosti, Angiology Department, Clinica MD Barbantini, via del Calcio, 55100 Lucca, Italy.

E-mail: jmosti@tin.it

Key words: graduated compression, progressive compression, inversely graduated compression, ejection fraction, venous pumping function.

Received for publication: 9 January 2014.

Revision received: 3 February 2014.

Accepted for publication: 3 February 2014.

This work is licensed under a Creative Commons Attribution 3.0 License (by-nc 3.0).

(C) Copyright G. Mosti, 2014

Licensee PAGEPress, Italy

Veins and Lymphatics 2014; 3:2235

doi:10.4081/vl.2014.2235

after manual compression of the calf, both at the junction of the GSV in the groin and $5 \mathrm{~cm}$ distally. Reflux time more than 1 second was considered to be pathological. Using a cross sectional view the diameter of the GSV was measured in the groin.

All individuals were informed about the investigation and gave their written consent. The reported studies complied with the Helsinki Declaration and the rules of the local ethical committee.

\section{Compression devices}

In different studies we used two different kinds of ready-made, knee length, elastic stockings and inelastic bandages.

All the compression devices were applied either to provide a standard graduated pressure profile or an inversely graduated or progressive pressure profile.

In the first study ${ }^{4}$ a standard graduated elastic compression stocking (GECS) was compared with a progressive elastic compression stocking (PECS). Both elastic stockings exerted a pressure at the gaiter area between 15 and $25 \mathrm{~mm} \mathrm{Hg}$. The GECS provided a degressive pressure profile, with pressures which were 20\% lower than at calf level (Progressivâ N'System, Pierre Fabre, Castres, France). The PECS (Pierre Fabre) exerted a lower pressure at the ankle and an increasing pressure profile with pressures about $50 \%$ higher at calf level than that at the ankle.

In the second study ${ }^{5}$ an inelastic multilayer, multicomponent bandage made up with Rosidal $\mathrm{K}^{\circledR}$ on top of a padding layer consisting of Cellona ${ }^{\circledR}$ synthetic cotton and Mollelast Haft $^{\circledR} \quad$ (Lohmann\&Rauscher, Rengsdorf, Germany), was wrapped on the lower leg from the base of the toe to the popliteal fossa. The standard application provided a higher pressure in the gaiter region and a decreasing 
pressure towards the calf [graduated compression bandages (GCB)]. This bandage was compared with a bandage applied with higher pressures over the calf than over the distal leg thereby producing a negative pressure gradient [negative graduated compression bandages (NGCB)].

In the third study, ${ }^{6}$ one PECS was compared with two PECS donned over each other and, subsequently, with one additional conventional stocking covering only the gaiter area to restore a graduate pressure profile.

\section{Interface pressure measurement}

In all studies the pressure exerted by different compression devices was continuously measured using a validated device ${ }^{7,8}$ connected with a data logger by a special computer program (Picopress ${ }^{\circledR}$, Microlabitalia, Padua, Italy). The pressure transducer consists of a flat plastic pressure probe (diameter $5 \mathrm{~cm}$ ) filled with $2 \mathrm{~mL}$ of air for the pressure measurement. Fluctuations of pressure on this probe are transformed into electronic signals (Statham-element) that can be recorded continuously. Two probes were used to measure pressure simultaneously: one at the distal leg, about $12 \mathrm{~cm}$ proximal to the medial malleolus (B1 point, which is defined by the transition of the muscular part of the medial gastrocnemius into the tendinous part) ${ }^{9}$ and one proximally, at the maximal calf circumference (C point). ${ }^{4}$ Interface pressure was measured continuously in the supine and the standing position and during the exercise program. Static stiffness index was calculated by subtracting supine pressure from standing pressure..$^{10}$

\section{Measurement of ejection fraction of the venous calf pump}

Using strain-gauge plethysmography (Angioflow2; Microlabitalia), ejection fraction was assessed following the method described by Poelkens et al. ${ }^{11}$ An indium-gallium alloy gauge (diameter of $1 \mathrm{~mm}$ ) is placed around the leg in the supine position $5 \mathrm{~cm}$ distally from the patella and immediately proximal to the upper border of the elastic stocking. After the calibration of the device, the investigation starts by elevating the examined leg in order to empty the veins and to record the minimal volume of the leg segment. After one minute the patient stands up and the volume increase of the calf segment encircled by the strain gauge probe, reflecting venous filling, is measured continuously. Venous volume (VV) is defined as the difference between empty and filled veins. During a standardized exercise (walking on spot with 20 steps in 20 seconds) the amount of blood that is expelled towards the heart [expelled volume (EV)] reflects the quality of the venous pump. Ejection fraction (\%) is calculated according to the formula $100 \times$
EV/VV. Using this method we were able to assess the hemodynamic efficacy of several compression products in a completely noninvasive way. ${ }^{12-15}$

The experiments were carried out in baseline condition without compression and repeated with the compression stockings or the inelastic bandages.

In every circumstance the sequence of tests (without compression or with different types of compression) was randomized.

All tests on every patient were done on the same day with an interval of 15 min between each measurement. The measurements were performed 5 min after stocking or bandages application with the patient resting in the supine position in a quiet room with constant humidity and temperature.

\section{Statistical analysis}

In all the studies median values and interquartile ranges are given.

Non-parametric Friedman test with Dunn's multiple comparisons were used to compare the repeated measurements of EF under different compression systems with the baseline. The Spearman rank test was taken as a non- parametric method for quantifying correlations. Differences with a $\mathrm{P}<0.05$ were considered statistically significant.

The graphs and the statistically evaluations were generated by using Graph Pad Prism5 software (Graph Pad, San Diego, CA, USA).

\section{Results}

\section{Ejection fraction}

EF was always significantly increased by both elastic and inelastic compression devices. The so called negative graduated or progressive compression was significantly more effective than the graduated compression in increasing the ejection fraction, both when applied by means of elastic stockings and by inelastic bandages (Figure 1 A,B). One elastic stocking is not able to restore the normal value of $\mathrm{EF}$, which is higher than $60 \%$, while two stockings over each other restore EF in its normal range (Figure 1C). Maintaining the same pressure over the calf exerted by two progressive stockings and additionally increasing the pressure over the ankle in order to move from

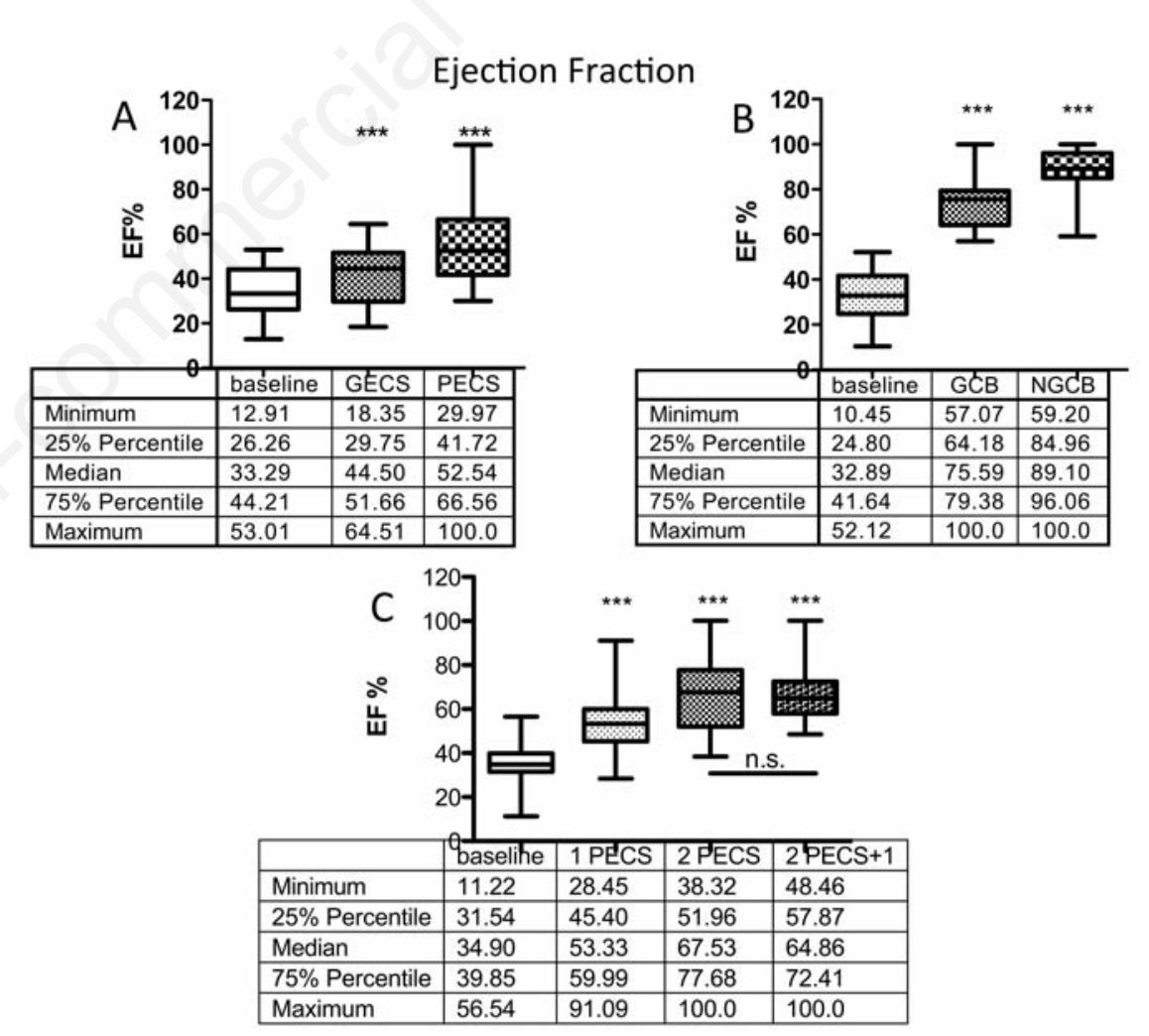

Figure 1. Ejection fraction in baseline conditions and with different compression devices. A) the low EF in baseline condition, increases with one GECS and increases significantly more with one PECS even if not EF was not restored into its normal range; $B$ ) the low EF in baseline conditions is restored into its by GCB, and even more with NGCB; C) one PECS increases EF, two PECS increases significantly more EF into its normal range; two PECS and a third stocking applied only on the gaiter area to restore a graduated pressure profile are not able to further increase EF. 
a progressive to a graduated pressure profile doesn't increase the ejection fraction (Figure 1C). In contrast to compression stockings inelastic bandages are able to normalize $\mathrm{EF}$ when applied with a graduated pressure profile. Inelastic bandages applied with a progressive pressure profile increase the EF significantly further, up into the range close to $90 \%$ (Figure 1B). The increase of EF is positively related to pressure and stiffness: increasing pressure and stiffness over the calf leads to progressively higher ejection fraction. ${ }^{4,5}$ The highest ejection fraction was recorded with inelastic bandages applied with a progressive pressure profile.

\section{Interface pressure}

In the first study comparing graduated and progressive elastic compression stockings, GECS exerted a higher pressure at ankle (median $22 \mathrm{~mm} \mathrm{Hg}$ ) and showed a decrease of the pressure by $14 \%$ at the calf (point C). PECS exerted a lower pressure at ankle level, median $18.5 \mathrm{~mm} \mathrm{Hg}$ and showed a pressure increase by $57 \%$ at $\mathrm{C}$ (Figure $2 \mathrm{~A}$ ).

These pressures increased only slightly by standing up and during exercise. The highest pressures were recorded with PECS at C point in standing position $(31.5 \mathrm{~mm} \mathrm{Hg})$, and during exercise $(32 \mathrm{~mm} \mathrm{Hg})$.

Comparing graduated and negatively graduated compression bandages, GCB median supine interface pressure was $53.5 \mathrm{~mm} \mathrm{Hg}$ at $\mathrm{B} 1$ and $37.5 \mathrm{~mm} \mathrm{Hg}$ at C. With NGCB median pressure was $50 \mathrm{~mm} \mathrm{Hg}$ at $\mathrm{Bl}$ and $62 \mathrm{~mm} \mathrm{Hg}$ at C (Figure 2B).

Standing pressures were significantly higher than supine pressures, both at position B1 and at $\mathrm{C}(\mathrm{P}<0.001)$ for both bandages. The greatest median pressure difference was in the calf area in standing position where GCB exerted $59 \mathrm{mmHg}$ and NGCB $75.5 \mathrm{mmHg}$ $(\mathrm{P}<0.001)$.

In the third study median compression pressure exerted by 2 superimposed stocking was significantly higher than with one stocking (33 vs $18 \mathrm{~mm} \mathrm{Hg}$ in $\mathrm{B} 1$ and 46 vs $27 \mathrm{~mm} \mathrm{Hg}$ in C) (Figure 2C). With 2 superimposed progressive stocking, maintaining the same pressure of 46 $\mathrm{mm} \mathrm{Hg}$ over the calf, we added a third stocking applied only over the foot and ankle region which was rolled down in order not to compress the calf. This increased the pressure over the ankle from 33 to $56 \mathrm{~mm} \mathrm{Hg}$ and restored a graduated pressure profile.

\section{Discussion}

Our data show that the higher the pressure over the calf and the higher the pressure peaks during walking, ${ }^{5}$ the grater the effect on venous pumping function: one PECS is more effective than one GECS, two PECS superimposed over each other are more effective than one PECS, NGCB is more effective than GCB. NGCB exerting the strongest pressure over the calf compared to other compression devices is the most effective modality. The reported data confirm that the pressure over the calf is mainly responsible for the venous pump improvement independently from the pressure gradient: maintaining the same pressure over the calf with a progressive pressure profile and increasing the pressure over the ankle to restore a graduated compression did not have any significant effect the venous pumping function.

In the standing position the intravenous hydrostatic pressure is maximal at foot and ankle level and progressively lower towards the upper leg and the thigh. In order to counteract this intravenous pressure profile the external pressure applied by compression stockings was postulated to be higher at ankle level with a continuous pressure reduction from distal to proximal. This graduated compression is considered an important quality criterion for compression systems and a cornerstone of standard care in venous disease.

Actually pressure peaks of more than 150 $\mathrm{mmHg}$ recorded in the solely muscle during walking ${ }^{16}$ producing inverse intravenous pressure gradients with every step are a physiolog- ical phenomenon. In addition simultaneous measurements revealed that ambulatory venous pressure decreases more in the foot than in the calf veins, both in normal and patients with superficial venous incompetence ${ }^{17}$ demonstrating that a continuous intravenous pressure gradient is not a general physiological principle during walking.

An intravenous pressure which is higher at calf than at ankle level could theoretically interfere with venous return. This is obviously not the case due to the complex interplay of contraction and relaxation of different leg muscle which produces a sequence of compressions and distensions of the leg veins favoring the venous outflow from the leg. In fact the first two phases of walking, plantar dorsi-flexion and plantar leaning of the foot, empty the distal foot and ankle veins into the calf deep system; during the third phase, ankle plantar flexion, the calf muscle contraction squeezes out the blood pooled in the full calf veins even producing a very short phase of distal blood pooling which will be emptied with the following step. ${ }^{18}$ As a consequence there is not a higher calf pressure at the same time as a lower ankle pressure but a sequence of different pressures favoring the venous emptying of the leg.

Therefore, when applying compression devices in the ambulatory patient affected by
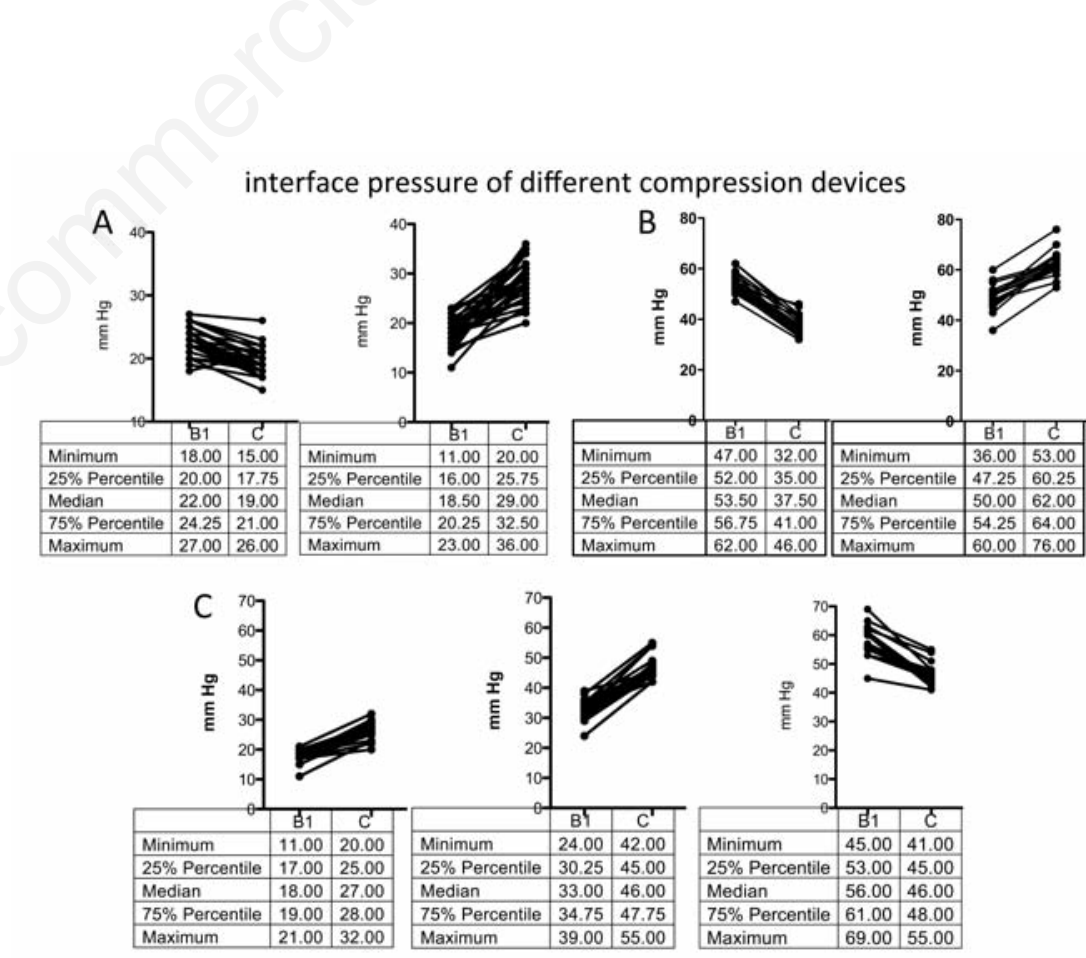

Figure 2. Pressure profile from ankle (B1) to calf (C) area of different compression devices. A) with GECS (left) pressure decreases from B to C, with one PECS (right) pressure increases from $B$ to $C$; $B$ ) GCB (left) produces a decreasing pressure from $B$ to $C$ while NGCB (right) produces an increasing pressure from $B$ to $C ; C$ ) one PECS increases pressure profile from B to C (left), two PECS (middle) shift to a higher level this pressure profile; 2 PECS plus a third stocking applied only on the gaiter area restore a graduated compression. 
chronic venous disorder, a graduated pressure profile cannot be considered mandatory. Actually compression devices exerting a progressive, pressure profile, higher on the calf than on the ankle have been proved to be more effective than graduated compression in increasing the venous pumping function assessed by measuring the EF from the lower leg. ${ }^{4-6}$

This effect can be explained by the higher external compression over the calf exerted by a progressive compression which will increase the pressure exerted on the local veins during muscle systole and will squeeze out more vigorously the great amount of blood pooled in the calf compared to the ankle area, which is covered by lower compression. In other words a negative graduated pressure profile provided by the progressive compression corresponds to the progressive intravenous pressure which is physiologically higher in the calf than in the ankle during muscle activity.

It can be argued that a strong pressure of about $50 \mathrm{~mm} \mathrm{Hg}$ or more over the calf (exerted by double PECS or progressive inelastic bandages) could impede the distal venous emptying of the foot and ankle veins into the calf veins. The greatest increase of EF recorded with the superimposition of two PECS or with the very strong pressure of the inelastic bandages demonstrates that this is not the case.

Finally compression comfort must be taken into account: a graduated compression with a high pressure over the calf would require a very strong pressure over the ankle, which can be intolerable; furthermore such a pressure profile could make donning very difficult when using an elastic stocking. This is not the case for progressive compression. ${ }^{6}$

The main limit of all these studies is that the benefit of progressive stockings on venous pumping function in patients with superficial veins incompetence cannot be extended to other clinical indications like edema, superficial or deep vein thrombosis, post thrombotic syndrome, venous ulcers or compression after venous procedures. Before recommending progressive compression its effectiveness in these clinical conditions needs to be carefully assessed in new studies. Also the sparse data regarding the use of not graduated material for edema prevention ${ }^{19}$ and of foot-sparing compression after varicose vein surgery ${ }^{20}$ should be confirmed in future trials.

\section{Conclusions}

Negatively graduated or progressive compression devices are significantly more effective than graduated compression in increasing ejection fraction up to restoring its normal range in patients with chronic venous disorders. Taking into account the reported experimental data a graduated pressure profile should not be considered as mandatory at least in ambulatory patients.

At present time other objective hemodynamic data as well as clinical outcomes concerning treatment or prevention of other venous clinical conditions are not available and should be investigated in future trials.

\section{References}

1. Couzan S, Leizorovicz A, Laporte S, et al. A randomized double-blind trial of upward progressive versus degressive compressive stockings in patients with moderate to severe chronic venous insufficiency. J Vasc Surg 2012;56:1344-50.

2. Couzan S, Assante C, Laporte S, et al. Booster study: comparative evaluation of a new concept of elastic stockings in mild venous insufficiency. Presse Med 2009;38: 355-61.

3. Garreau C, Pibourdin JM, Nguyen Le C, Boisseau MR. Elastic compression in golf competition. J Mal Vasc 2008;33:250-1.

4. Mosti G, Partsch H. Compression stockings with a negative pressure gradient have a more pronounced effect on venous pumping function than graduated elastic compression stockings. Eur J Vasc Endovasc Surg 2011;42:261-6.

5. Mosti G, Partsch H. High compression pressure over the calf is more effective than graduated compression in enhancing venous pump function. Eur J Vasc Endovasc Surg 2012;44:332-6.

6. Mosti G. Partsch H. Improvement of venous pumping function by double progressive compression stockings: higher pressure over the calf is more important than graduated pressure profile. Eur J Vasc Endovasc Surg 2014 [In Press].

7. Mosti G., Rossari S. L'importanza della misurazione della pressione sottobendaggio e presentazione di un nuovo strumento di misura. Acta Vulnol 2008;6:31-6.

8. Partsch H, Mosti G. Comparison of three portable instruments to measure compression pressure. Int Angiol 2010;29:426-30.

9. Partsch H, Clark M, Bassez S, et al. Measurement of lower leg compression in vivo: recommendations for the performance of measurements of interface pressure and stiffness. Dermatol Surg 2006;32: 224-33.

10. Partsch H. The static stiffness index: a simple method to assess the elastic property of compression material in vivo. Dermatol Surg 2005;31:625-30.

11. Poelkens F, Thijssen DH, Kersten B, et al. Counteracting venous stasis during acute lower leg immobilization. Acta Physiol 2006;186:111-8.

12. Mosti G, Partsch H. Measuring venous pumping function by strain-gauge plethysmography. Int Angiol 2010;29:421-5.

13. Mosti G, Mattaliano V, Partsch H. Inelastic compression increases venous ejection fraction more than elastic bandages in patients with superficial venous reflux. Phlebology 2008;23:287-94.

14. Mosti G, Partsch H. Is low compression pressure able to improve venous pumping function in patients with venous insufficiency? Phlebology 2010;25:145-50.

15. Mosti G, Partsch H. Inelastic bandages maintain their hemodynamic effectiveness over time despite significant pressure loss. J Vasc Surg 2010;52:925-31.

16. Murthy G, Ballard RE, Breit GA, Watenpaugh DE, Hargens AR. Intramuscular pressures beneath elastic and inelastic leggings. Ann Vasc Surg 1994;8: 543-8.

17. Stranden E, Ogreid P, Seem E. Venous pressure gradients in patients with chronic venous disease. Phlebology 1986;1:47-50.

18. Gardner AMN, Fox RH. The venous system in health and disease. IOS Press, The Netherlands; 2001. pp 42-73.

19. Mosti G, Partsch H. Occupational leg oedema is more reduced by antigraduated than by graduated stockings. Eur J Vasc Endovasc Surg 2013;45:523-7.

20. Ricci S, Moro L, Trillo L, Incalzi RA. Footsparing postoperative compression bandage: a possible alternative to the traditional bandage. Phlebology 2013;28:47-50. 